PROCEEDINGS OF THE

AMERICAN MATHEMATICAL SOCIETY

Volume 136, Number 12, December 2008, Pages 4157-4165

S 0002-9939(08)09543-9

Article electronically published on June 20, 2008

\title{
ON ENUMERATION OF CONJUGACY CLASSES OF COXETER ELEMENTS
}

\author{
MATTHEW MACAULEY AND HENNING S. MORTVEIT
}

(Communicated by Jim Haglund)

\begin{abstract}
In this paper we study the equivalence relation on the set of acyclic orientations of a graph $Y$ that arises through source-to-sink conversions. This source-to-sink conversion encodes, e.g. conjugation of Coxeter elements of a Coxeter group. We give a direct proof of a recursion for the number of equivalence classes of this relation for an arbitrary graph $Y$ using edge deletion and edge contraction of non-bridge edges. We conclude by showing how this result may also be obtained through an evaluation of the Tutte polynomial as $T_{Y}(1,0)$, and we provide bijections to two other classes of acyclic orientations that are known to be counted in the same way. A transversal of the set of equivalence classes is given.
\end{abstract}

\section{INTRODUCTION}

The equivalence relation on the set of acyclic orientations of a graph $Y$ that arises from iteratively changing sources into sinks appears in many areas of mathematics. For example, in the context of Coxeter groups the source-to-sink operation encodes conjugation of Coxeter elements 11, although in general these conjugacy classes are not fully understood. Additionally, it is closely related to the reflection functor in the representation theory of quivers 6 . It has also been studied in the context of the chip-firing game of Björner, Lovász, and Shor [1]. Moreover, it arises in the characterization of cycle equivalence for a class of discrete dynamical systems [5], which was the original motivation for this work.

In [11, the number of equivalence classes $\kappa(Y)$ for a graph $Y$ was determined for graphs that contain precisely one cycle. The main result of this paper is a novel proof for $\kappa(Y)$ for arbitrary graphs in the form of a recurrence relation involving the edge deletion $Y_{e}^{\prime}$ and edge contraction $Y_{e}^{\prime \prime}$ of a cycle-edge $e$ in $Y$. It can be stated as follows.

Theorem 1.1. Let e be a cycle-edge of $Y$. Then

$$
\kappa(Y)=\kappa\left(Y_{e}^{\prime}\right)+\kappa\left(Y_{e}^{\prime \prime}\right) .
$$

Our proof involves a careful consideration of what happens to the $\kappa$-equivalence classes of $\operatorname{Acyc}(Y)$ as a cycle-edge $e$ is deleted. This leads to the construction of the collapse graph of $Y$ and $e$, which has as vertex set the $\kappa$-classes of $\operatorname{Acyc}(Y)$. We show that there is a bijection from the set of connected components of this graph to the

Received by the editors November 7, 2007.

2000 Mathematics Subject Classification. Primary 20F55, 05A99, 06A06.

(C)2008 American Mathematical Society 
set of $\kappa$-equivalence classes of $\operatorname{Acyc}\left(Y_{e}^{\prime}\right)$. Moreover, we establish a bijection from the edge set of the collapse graph to the set of $\kappa$-equivalence classes of $\operatorname{Acyc}\left(Y_{e}^{\prime \prime}\right)$. From this and the fact that the collapse graph is a forest, the recursion (1.1) follows. Alternatively, the recursion can be derived through an observation made by Vic Reiner (see [7, Remark 5.5]): the number of equivalence classes of linear orderings under the operations of $(i)$ transposition of successive, non-connected generators and (ii) cyclic shifts is counted by (1.1). The bijection between Coxeter elements and acyclic orientations in [11] provides the connection to our setting. Even though the connection of this fact to the enumeration of conjugacy classes of Coxeter elements is straightforward, it does not appear in the literature. Our contribution is an independent and direct proof of this result by examining the acyclic orientations of the Coxeter graph. Additionally, our proof provides insight into the structure of the equivalence classes. We believe that the techniques involved may be useful in extending current results in Coxeter theory, in particular, some from [11.

Let $Y$ be a finite undirected graph with vertex set $\mathrm{v}[Y]=\{1,2, \ldots, n\}$ and edge set $\mathrm{e}[Y]$. An orientation of $Y$ is represented by a map $O_{Y}: \mathrm{e}[Y] \longrightarrow \mathrm{v}[Y] \times \mathrm{v}[Y]$, and the graph $G\left(O_{Y}\right)$ is obtained from $Y$ by orienting each edge as given by $O_{Y}$. We will use $O_{Y}$ and $G\left(O_{Y}\right)$ interchangeably when no ambiguity can arise. An orientation $O_{Y}$ is acyclic if $G\left(O_{Y}\right)$ has no directed cycles. The set of acyclic orientations of $Y$ is denoted by $\operatorname{Acyc}(Y)$, and we set $\alpha(Y)=|\operatorname{Acyc}(Y)|$, which can be computed through the well-known recursion relation

$$
\alpha(Y)=\alpha\left(Y_{e}^{\prime}\right)+\alpha\left(Y_{e}^{\prime \prime}\right) .
$$

As above, $Y_{e}^{\prime}$ and $Y_{e}^{\prime \prime}$ are the graphs obtained from $Y$ by deletion and contraction of a fixed edge $e$, respectively. For the results in this paper we may without loss of generality assume that $\mathrm{v}[Y]$ is linearly ordered. Let $e=\{v, w\}$ and assume $v<w$. The graph $Y_{e}^{\prime}$ has the same vertex set as $Y$ and the edge set $\mathrm{e}[Y] \backslash\{e\}$. The graph $Y_{e}^{\prime \prime}$ has the vertex set $\mathrm{v}[Y] \backslash\{w\}$. Its edge set consists of all edges of $Y$ not incident with $w$ and with an edge $\{u, v\}$ added for each edge $e^{\prime}=\{u, w\} \neq e$ of $Y$ such that $\{u, v\} \notin \mathrm{e}[Y]$. Thus if $Y$ is simple and loop-free, then so is $Y_{e}^{\prime \prime}$. It is known that there is a bijection between $\operatorname{Acyc}(Y)$ and the set of Coxeter elements of the Coxeter group whose Coxeter graph is $Y$ [4, 10]. There is also a bijection between $\operatorname{Acyc}(Y)$ and the set of chambers of the graphic hyperplane arrangement $\mathcal{H}(Y)[8]$.

If $v$ is a source of an acyclic orientation $O_{Y}$ with degree $\geq 1$, then reversing the orientation of all the edges incident to $v$ maps $O_{Y}$ to a new orientation of $Y$, which is also acyclic. This is called a source-to-sink operation, or a click. We define the equivalence relation $\sim_{\kappa}$ on the set of acyclic orientations for a fixed graph $Y$ by $O_{Y} \sim_{\kappa} O_{Y}^{\prime}$ if there is a sequence of source-to-sink operations that maps $O_{Y}$ to $O_{Y}^{\prime}$. Two such orientations are said to be click-equivalent, or $\kappa$-equivalent. We set $\kappa(Y)=\left|\operatorname{Acyc}(Y) / \sim_{\kappa}\right|$.

The set of linear orders on $\mathrm{v}[Y]$ can be represented by the set of permutations of v $[Y]$, which we denote as $S_{Y}$. Note that $\pi \in S_{Y}$ induces an acyclic orientation $O_{Y}^{\pi}$ of $Y$ by $O_{Y}^{\pi}(\{i, j\})=(i, j)$ if $i$ precedes $j$ in $\pi$, and $O_{Y}^{\pi}(\{i, j\})=(j, i)$ otherwise. We write $[\pi]_{Y}$ for the set of linear orders compatible with the acyclic orientation $O_{Y}$ induced by $\pi$. There is a bijection between $\left\{[\pi]_{Y} \mid \pi \in S_{Y}\right\}$ and $\operatorname{Acyc}(Y)$; see e.g. 9. Let $\pi$ be the permutation representation of a linear order compatible with $O_{Y}$. Note that mapping $\pi=\left(\pi_{1}, \pi_{2}, \ldots, \pi_{n}\right)$ to $\pi^{\prime}=\left(\pi_{2}, \ldots, \pi_{n}, \pi_{1}\right)$ corresponds to converting $\pi_{1}$ from a source to a sink in $O_{Y}$. In general, two distinct acyclic orientations $O_{Y}$ and $O_{Y}^{\prime}$ are $\kappa$-equivalent if and only if there exist $\pi$ compatible 
with $O_{Y}$ and $\pi^{\prime}$ compatible with $O_{Y}^{\prime}$ such that $\pi^{\prime}$ can be obtained from $\pi$ by $(i)$ cyclic shifts and (ii) transpositions of consecutive elements that are not connected in $Y$. For a given Coxeter group $W$ with generators $S=\left\{s_{i}\right\}_{i=1}^{n}$ and Coxeter graph $Y$, there is a similar mapping from $S_{Y}$ into the set of Coxeter elements $C(W)$ and a bijection from $C(W)$ to $\operatorname{Acyc}(Y)$; see [10]. Thus, an acyclic orientation represents a unique Coxeter element, and a source-to-sink operation corresponds to conjugating that element by a particular generator. Therefore, $\kappa(Y)$ is an upper bound for the number of conjugacy classes of Coxeter elements in a Coxeter group whose Coxeter graph is $Y$, and this bound is known to be sharp in certain cases [11. A simple induction argument shows that if $Y$ is a tree, then $\kappa(Y)=1$, and thus all Coxeter elements in a finite Coxeter group are conjugate [4. In 11, the author shows that if $Y$ contains a single cycle of length $n$, then $\kappa(Y)=n-1$. This becomes a straightforward corollary of Theorem 1.1. The recurrence of Theorem 1.1 appears in several areas of mathematics and corresponds to the evaluation of the Tutte polynomial at $(1,0)$, which we describe in Section 4 .

\section{Preliminary Results}

We begin our study of $\kappa(Y)$ by making the following simple observation recorded without proof.

Proposition 2.1. Let $Y$ be the disjoint union of undirected graphs $Y_{1}$ and $Y_{2}$. Then

$$
\kappa(Y)=\kappa\left(Y_{1}\right) \kappa\left(Y_{2}\right) .
$$

In light of this, we may assume that $Y$ is connected when computing $\kappa(Y)$. An edge of $Y$ that is not contained in any simple cycle of $Y$ is a bridge; otherwise it is a cycle-edge. The graph obtained from $Y$ by deletion of all bridges is the cycle graph of $Y$, and it is denoted by $\operatorname{Cycle}(Y)$. Alternatively, an edge $e$ of a connected graph $Y$ is a bridge if the deletion of $e$ disconnects $Y$. Bridges do not contribute to $\kappa(Y)$, as shown in the following proposition.

Proposition 2.2. Let $Y$ be an undirected graph, and let $e=\{v, w\}$ be a bridge of $Y$, connecting the disjoint subgraphs $Y_{1}$ and $Y_{2}$. Then one has the relation

$$
\kappa(Y)=\kappa\left(Y_{1}\right) \kappa\left(Y_{2}\right) .
$$

Proof. Each pair of acyclic orientations $O_{Y_{1}} \in \operatorname{Acyc}\left(Y_{1}\right)$ and $O_{Y_{2}} \in \operatorname{Acyc}\left(Y_{2}\right)$ extends to exactly two acyclic orientations of $Y$ by $O_{Y}=\left(O_{Y_{1}},(v, w), O_{Y_{2}}\right)$ and $O_{Y}^{\prime}=\left(O_{Y_{1}},(w, v), O_{Y_{2}}\right)$ defined in the obvious way. Clearly, every acyclic orientation of $Y$ is also of one of these forms. Moreover, any click sequence for $O_{Y}^{\prime}$ that contains each vertex of $Y_{2}$ exactly once and contains no vertex of $Y_{1}$ maps $O_{Y}^{\prime}$ to $O_{Y}$. Hence $O_{Y}$ and $O_{Y}^{\prime}$ are click-equivalent. It follows that $O_{Y}, O_{Y}^{\prime} \in \operatorname{Acyc}(Y)$ are click-equivalent if and only their corresponding acyclic orientations over $Y_{1}$ and $Y_{2}$ are click-equivalent, and the equality (2.2) now follows from Proposition 2.1.

Proposition 2.2 gives us an immediate corollary.

Corollary 2.3. For any undirected graph $Y$ we have $\kappa(Y)=\kappa(\operatorname{Cycle}(Y))$. In particular, if $Y$ is a forest, then $\kappa(Y)=1$.

We remark that the first part of this corollary is proven in [11 for the special case where $\operatorname{Cycle}(Y)$ is a circle. The second part is well-known (see e.g. (4)). 
Let $P=\left(v_{1}, v_{2}, \ldots, v_{k}\right)$ be a (possibly closed) simple path in $Y$. The map

$$
\nu_{P}: \operatorname{Acyc}(Y) \longrightarrow \mathbb{Z}
$$

evaluated at $O_{Y}$ is the number of edges of the form $\left\{v_{i}, v_{i+1}\right\}$ in $Y$ oriented as $\left(v_{i}, v_{i+1}\right)$ in $O_{Y}$ (positive edges) minus the number of edges oriented as $\left(v_{i+1}, v_{i}\right)$ in $O_{Y}$ (negative edges).

Lemma 2.4. Let $P$ be a simple closed path in the undirected graph $Y$. The map $\nu_{P}$ induces a map $\nu_{P}^{*}$ : $\operatorname{Acyc}(Y) / \sim_{\kappa} \longrightarrow \mathbb{Z}$.

Proof. Let $c\left(O_{Y}\right)=O_{Y}^{\prime}$, where $c=c_{v}$ is a click of a single vertex $v$. If $v$ is not an element of $P$, then clearly $\nu_{P}\left(O_{Y}\right)=\nu_{P}\left(O_{Y}^{\prime}\right)$. On the other hand, if $v$ is contained in $P$, then $c$ maps one positive edge into a negative edge and vice versa. The general case follows by induction on the length of the click-sequence.

Lemma 2.4 will be used extensively in the proof of the main result in the next section.

\section{Proof of the MAin THEOREM}

From Proposition 2.2 it is clear that for the computation of $\kappa(Y)$ all bridges may be omitted. We now turn our attention to the role played by cycle-edges in determining $\kappa(Y)$ and to the proof of the recursion relation

$$
\kappa(Y)=\kappa\left(Y_{e}^{\prime}\right)+\kappa\left(Y_{e}^{\prime \prime}\right)
$$

of Theorem 1.1 valid for any cycle-edge $e$ of $Y$. We set $e=\{v, w\}$ in the following.

First, define $\iota_{1}: \operatorname{Acyc}\left(Y_{e}^{\prime \prime}\right) \longrightarrow \operatorname{Acyc}(Y)$ to be the map that sends $O_{Y^{\prime \prime}} \in \operatorname{Acyc}\left(Y_{e}^{\prime \prime}\right)$ to $O_{Y} \in \operatorname{Acyc}(Y)$ for which $O_{Y}(e)=(v, w)$ and for which all other edge orientations are inherited. The map $\iota_{2}: \operatorname{Acyc}\left(Y_{e}^{\prime \prime}\right) \longrightarrow \operatorname{Acyc}(Y)$ is defined analogously but orients $e$ to be $(w, v)$.

Proposition 3.1. The maps $\iota_{1}, \iota_{2}: \operatorname{Acyc}\left(Y_{e}^{\prime \prime}\right) \longrightarrow \operatorname{Acyc}(Y)$ extend to well-defined maps

$$
\iota_{1}^{*}, \iota_{2}^{*}: \operatorname{Acyc}\left(Y_{e}^{\prime \prime}\right) / \sim_{\kappa} \longrightarrow \operatorname{Acyc}(Y) / \sim_{\kappa} .
$$

Proof. For $\phi \in\left\{\iota_{1}, \iota_{2}\right\}$ and for any click-sequence $c$ of $O_{Y^{\prime \prime}} \in \operatorname{Acyc}\left(Y^{\prime \prime}\right)$ we have the commutative diagram

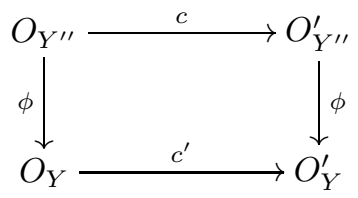

where the click-sequence $c^{\prime}$ over $Y$ is constructed from the click-sequence $c$ over $Y^{\prime \prime}$ by insertion of $w$ after (resp. before) every occurrence of $v$ in $c$ for $\iota_{1}$ (resp. $\iota_{2}$ ).

Proposition 3.2. Let e be a cycle-edge. For any $\left[O_{Y^{\prime \prime}}\right] \in \operatorname{Acyc}\left(Y^{\prime \prime}\right)$ we have $\iota_{1}^{*}\left(\left[O_{Y^{\prime \prime}}\right]\right) \neq \iota_{2}^{*}\left(\left[O_{Y^{\prime \prime}}\right]\right)$.

Proof. Let $P$ be any simple closed path containing $e$ and oriented so as to include $(v, w)$. From the definition of $\iota_{1}$ and $\iota_{2}$ we conclude that $\nu_{P}\left(\iota_{1}\left(O_{Y^{\prime \prime}}\right)\right)=$ $\nu_{P}\left(\iota_{2}\left(O_{Y^{\prime \prime}}\right)\right)+2$, and the proposition follows by Lemma 2.4.

Proposition 3.3. Both maps $\iota_{1}^{*}$ and $\iota_{2}^{*}$ are injective. 
Proof. We prove the statement for $\iota_{1}^{*}$. The proof for $\iota_{2}^{*}$ is analogous. Assume $\left[O_{Y^{\prime \prime}}\right] \chi_{\kappa}\left[O_{Y^{\prime \prime}}^{\prime}\right]$ both are mapped to $\left[O_{Y}\right]$ under $\iota_{1}^{*}$. By construction, any elements $O_{Y^{\prime \prime}}$ and $O_{Y^{\prime \prime}}^{\prime}$ of the respective $\kappa$-classes have $\iota_{1}$-images with $e$ oriented as $(v, w)$. Moreover, for any $O_{Y}$ in the image of $\iota_{1}$ there is no directed path from $v$ to $w$ of length $\geq 2$, and there is no directed path from $w$ to $v$. By a suitable choice of representatives we may also assume that $v$ is a source in both $O_{Y^{\prime \prime}}$ and $O_{Y^{\prime \prime}}^{\prime}$. From this it is clear that $v$ and $w$ belong to successive layers in the acyclic orientations $O_{Y}$ and $O_{Y}^{\prime}$.

Let $c$ be a click-sequence taking $\iota_{1}\left(O_{Y^{\prime \prime}}\right)$ to $\iota_{1}\left(O_{Y^{\prime \prime}}^{\prime}\right)$. Again by construction, we may assume that any occurrence of $v$ in $c$ is immediately followed by $w$. This follows since $v$ and $w$ have to occur equally many times in $c$, and from the fact that $v$ and $w$ belong to successive layers in $O_{Y}$ and $O_{Y}^{\prime}$. If $v$ and $w$ were not consecutive in $c$, it could only be because $c$ is of the form $c=\ldots v v_{1} \ldots v_{k} w_{1} \ldots w_{r} w \ldots$, where the $v_{i}$ 's belong to the same layer as $v$ and the $w_{i}$ 's belong to the same layer as $w$. A layer is in particular an independent set, and it is clear that the sequence $c^{\prime}=\ldots v_{1} \ldots v_{k} v w w_{1} \ldots w_{r} \ldots$ obtained from $c$ by changing the order of $v$ and $w$ also maps $O_{Y}$ to $O_{Y}^{\prime}$.

The click-sequence $c^{\prime \prime}$ obtained from $c^{\prime}$ by deleting every occurrence of $w$ is a click-sequence mapping $O_{Y^{\prime \prime}}$ to $O_{Y^{\prime \prime}}^{\prime}$, which contradicts the assumption that $\left[O_{Y^{\prime \prime}}\right] \nsim_{\kappa}\left[O_{Y^{\prime \prime}}^{\prime}\right]$.

Consequently, any $\kappa$-class $\left[O_{Y}\right]$ contains at most one set of the form $\iota_{1}\left(\left[O_{Y^{\prime \prime}}\right]\right)$ and at most one set of the form $\iota_{2}\left(\left[O_{Y^{\prime \prime}}\right]\right)$.

Proposition 3.4. Let e be a cycle-edge of the undirected graph $Y$. For each pair of distinct $\kappa$-classes $\left[O_{Y}\right]$ and $\left[O_{Y}^{\prime}\right]$ there is at most one $\kappa$-class $\left[O_{Y^{\prime \prime}}\right]$ such that $\left\{\iota_{1}^{*}\left(\left[O_{Y^{\prime \prime}}\right]\right), \iota_{2}^{*}\left(\left[O_{Y^{\prime \prime}}\right]\right)\right\}=\left\{\left[O_{Y}\right],\left[O_{Y}^{\prime}\right]\right\}$.

Proof. Assume that this is not the case and that there in fact is another class $\left[O_{Y^{\prime \prime}}^{\prime}\right]$ with the same property. Since both maps $\iota_{1}^{*}$ and $\iota_{2}^{*}$ are injective, it then follows (up to relabeling) that $\iota_{1}^{*}\left(\left[O_{Y^{\prime \prime}}\right]\right)=\iota_{2}^{*}\left(\left[O_{Y^{\prime \prime}}^{\prime}\right]\right)=\left[O_{Y}\right]$ and $\iota_{1}^{*}\left(\left[O_{Y^{\prime \prime}}^{\prime}\right]\right)=\iota_{2}^{*}\left(\left[O_{Y^{\prime \prime}}\right]\right)=$ $\left[O_{Y}^{\prime}\right]$. By the same argument as that in the proof of Proposition 3.2 it follows by using $\left[O_{Y^{\prime \prime}}\right]$ that $\nu_{P}^{*}\left(\left[O_{Y}\right]\right)=\nu_{P}^{*}\left(\left[O_{Y}^{\prime}\right]\right)+2$. On the other hand, by using $\left[O_{Y^{\prime \prime}}^{\prime}\right]$ it follows that $\nu_{P}^{*}\left(\left[O_{Y}^{\prime}\right]\right)=\nu_{P}^{*}\left(\left[O_{Y}\right]\right)+2$, which is impossible.

Definition 3.5. Let $e$ be a cycle-edge of the undirected graph $Y$. The collapse graph $\mathfrak{C}_{e}(Y)$ of $Y$ and $e$ is the graph with a vertex set $\operatorname{Acyc}(Y) / \sim_{\kappa}$ and edge set $\left\{\left\{\iota_{1}^{*}\left(\left[O_{Y^{\prime \prime}}\right]\right), \iota_{2}^{*}\left(\left[O_{Y^{\prime \prime}}\right]\right)\right\} \mid\left[O_{Y^{\prime \prime}}\right] \in \operatorname{Acyc}\left(Y^{\prime \prime}\right) / \sim_{\kappa}\right\}$.

Note that by Proposition 3.4 the graph $\mathfrak{C}_{e}(Y)$ is simple, and by Proposition 3.2 . it has no loops.

A line graph on $k$ vertices has vertices $1,2, \ldots, k$ and edges $\{i, i+1\}$ for $1 \leq i \leq$ $k-1$.

Proposition 3.6. Let e be a cycle-edge of the undirected graph $Y$. The collapse graph $\mathfrak{C}_{e}(Y)$ is isomorphic to a disjoint collection of line graphs.

Proof. By Definition 3.5 and the remark following it, each vertex in the collapse graph has degree $\leq 2$. Thus it is sufficient to show that $\mathfrak{C}_{e}(Y)$ contains no cycles. Let $P$ be a simple closed path in $Y$ containing the edge $e$. Then two adjacent $\kappa$-classes in $\mathfrak{C}_{e}(Y)$ differ in their $\nu_{P}^{*}$-values by precisely 2 . Assume $\mathfrak{C}_{e}(Y)$ contains 
the subgraph (up to relabeling)

$$
\begin{array}{ccc}
A^{\prime \prime} & A & A^{\prime}, \\
\iota_{1}^{*}\left(A_{1}\right) & \iota_{2}^{*}\left(A_{1}\right)=\iota_{1}^{*}\left(A_{2}\right) & \iota_{2}^{*}\left(A_{2}\right)
\end{array}
$$

for unique $\kappa$-classes $A_{1}, A_{2} \in \operatorname{Acyc}\left(Y^{\prime \prime}\right) / \sim_{\kappa}$. Clearly $\nu_{P}^{*}\left(A^{\prime \prime}\right)=\nu_{P}^{*}(A)+2$ and $\nu_{P}^{*}\left(A^{\prime}\right)=\nu_{P}^{*}(A)-2$. We now have the following situation: $(i)$ the $\nu^{*}$-values of adjacent vertices in $\mathfrak{C}_{e}(Y)$ differ by precisely 2 , and $(i i)$ the value of $\nu^{*}$ increases by 2 across each edge in the $A^{\prime \prime}$-direction relative to $A$ and decreases by 2 across each edge in the $A^{\prime}$-direction relative to $A$. If the subgraph in (3.4) was a part of a cycle of length $\geq 3$ in $\mathfrak{C}_{e}(Y)$, then by $(i i)$ there must be some pair of adjacent vertices for which $\nu^{*}$ differs by at least 4 . But this is impossible by $(i)$; hence $\mathfrak{C}_{e}(Y)$ contains no cycles, and the proof is complete.

Proposition 3.7. Let e be a cycle-edge of the undirected graph $Y$. Then $\kappa$-classes on the same connected component in $\mathfrak{C}_{e}(Y)$ map to the same $\kappa$-class in $\operatorname{Acyc}\left(Y^{\prime}\right) / \sim_{\kappa}$ upon the deletion of $e$.

Proof. It is sufficient to show this for adjacent vertices in $\mathfrak{C}_{e}(Y)$ - the general result follows by induction. Clearly, $O_{Y} \sim_{\kappa} O_{Y}^{\prime}$ implies $O_{Y^{\prime}} \sim_{\kappa} O_{Y^{\prime}}^{\prime}$. Adjacent vertices in $\mathfrak{C}_{e}(Y)$ contain elements that only differ in their orientations of $e$ and hence become $\kappa$-equivalent upon the deletion of $e$. The result follows.

Proposition 3.8. There is a bijection between the connected components in $\mathfrak{C}_{e}(Y)$ and $\operatorname{Acyc}\left(Y_{e}^{\prime}\right) / \sim_{\kappa}$.

Proof. Let $n_{c}$ denote the number of connected components of $\mathfrak{C}_{e}(Y)$. By Proposition 3.7 if $\left[O_{Y}\right]$ and $\left[O_{Y}^{\prime}\right]$ are connected in $\mathfrak{C}_{e}(Y)$, then both classes are contained in the same $\kappa$-class over $Y^{\prime}$, and thus $n_{c} \leq \kappa\left(Y^{\prime}\right)$.

It is clear that a $\kappa$-class contains all acyclic orientations for which there are representative permutations that are related by a sequence of adjacent transpositions of non-connected vertices in $Y$ and cyclic shifts. Upon deletion of the cycle-edge $e$ the adjacent transposition of the endpoints of $e$ becomes permissible, and thus two distinct $\kappa$-classes in $Y$ containing acyclic orientations that only differ on $e$ are contained within the same $\kappa$-class over $Y^{\prime}$. By reference to the underlying permutations, it follows that two $\kappa$-classes in $Y$ are contained within the same $\kappa$-class in $Y^{\prime}$ if and only if there is a sequence of $\kappa$-classes in $Y$ where consecutive elements in the sequence contain acyclic orientations that differ precisely on $e$. By the definition of $\mathfrak{C}_{e}(Y)$ it follows that all $\kappa$-classes over $Y$ that merge to be contained within one $\kappa$-class in $Y^{\prime}$ upon deletion of $e$ are contained within the same connected component of $\mathfrak{C}_{e}(Y)$, and thus $n_{c} \geq \kappa\left(Y^{\prime}\right)$.

Proof of Theorem 1.1. Upon the deletion of $e$ in $Y$, two or more $\kappa$-classes over $Y$ may merge to be contained with the same $\kappa$-class over $Y^{\prime}$. By Proposition 3.8 the number of $\kappa$-classes over $Y^{\prime}$ equals the number of connected components in $\mathfrak{C}_{e}(Y)$. If a connected component in $\mathfrak{C}_{e}(Y)$ contains $m$ distinct $\kappa$-classes of $Y$, then by Proposition 3.4 there are $m-1$ unique corresponding $\kappa$-classes over $Y^{\prime \prime}$. Thus for each component of $\mathfrak{C}_{e}(Y)$ we have a relation precisely of the form (1.1) for the $\kappa$-classes involved. The theorem now follows. 


\section{Related ENUMERATION PROBLEMS}

In this section we relate the problem of computing $\kappa(Y)=\left|\operatorname{Acyc}(Y) / \sim_{\kappa}\right|$ to two other enumeration problems where the same recurrence holds. We will show how these problems are equivalent and, additionally, how they all can be computed through an evaluation of the Tutte polynomial. As a corollary we obtain a transversal of $\operatorname{Acyc}(Y) / \sim_{\kappa}$.

In 2] the notion of cut-equivalence of acyclic orientations is studied. Recall that a cut of a graph $Y$ is a partition of the vertex set into two classes, $\mathrm{v}[Y]=V_{1} \sqcup V_{2}$, and where $\left[V_{1}, V_{2}\right]$ is the set of (cut-)edges between $V_{1}$ and $V_{2}$. A cut of a directed graph of the form $G\left(O_{Y}\right)$, which we simply refer to as a cut of $O_{Y}$, is oriented with respect to $O_{Y}$ if the edges of $\left[V_{1}, V_{2}\right]$ are all directed from $V_{1}$ to $V_{2}$ or are all directed from $V_{2}$ to $V_{1}$.

Definition 4.1. Two acyclic orientations $O_{Y}$ and $O_{Y}^{\prime}$ are cut-equivalent if the set $\left\{e \in \mathrm{v}[Y] \mid O_{Y}(e) \neq O_{Y}^{\prime}(e)\right\}$ is empty or is an oriented cut with respect to either $O_{Y}$ or $O_{Y}^{\prime}$.

The study of cut-equivalence in [2] was done outside the setting of Coxeter theory, and here we provide the connection.

Proposition 4.2. Two acyclic orientations of $Y$ are $\kappa$-equivalent if and only if they are cut-equivalent.

Proof. Suppose that distinct elements $O_{Y}$ and $O_{Y}^{\prime}$ in $\operatorname{Acyc}(Y)$ are cut-equivalent and, without loss of generality, that all edges of $\left[V_{1}, V_{2}\right]$ are oriented from $V_{1}$ to $V_{2}$ in $O_{Y}$. A click-sequence containing each vertex of $V_{1}$ precisely once maps $O_{Y}$ to $O_{Y}^{\prime}$, thus $O_{Y} \sim_{\kappa} O_{Y}^{\prime}$.

Conversely, suppose that $O_{Y} \sim_{\kappa} O_{Y}^{\prime}$, where $O_{Y}^{\prime}$ is obtained from $O_{Y}$ by a clicksequence containing a single vertex $v$. Then $O_{Y}$ and $O_{Y}^{\prime}$ are cut-equivalent, with the partition being $\{v\} \sqcup \mathrm{v}[Y] \backslash\{v\}$.

Obviously, the recurrence relation in (1.1) holds for the enumeration of both cut-equivalence and $\kappa$-equivalence classes.

Definition 4.3. The Tutte polynomial of an undirected graph $Y$ is defined as follows. If $Y$ has $b$ bridges, $\ell$ loops, and no cycle-edges, then $T_{Y}(x, y)=x^{b} y^{\ell}$. If $e$ is a cycle-edge of $Y$, then

$$
T_{Y}(x, y)=T_{Y_{e}^{\prime}}(x, y)+T_{Y_{e}^{\prime \prime}}(x, y) .
$$

We remark that it is well-known that the number of acyclic orientations of a graph $Y$ can be evaluated as $\alpha(Y)=T_{Y}(2,0)$. It was shown in 2] that the number of cut-equivalence classes can be computed through an evaluation of the Tutte polynomial as $T_{Y}(1,0)$, and thus $\kappa(Y)=T_{Y}(1,0)$.

It is known that $T_{Y}(1,0)$ counts several other quantities, some of which can be found in [3]. One of these is $\left|\operatorname{Acyc}_{v}(Y)\right|$, the number of acyclic orientations of $Y$ where a fixed vertex $v$ is the unique source. As the next result shows, there is a bijection between $\operatorname{Acyc}(Y) / \sim_{\kappa}$ and $\operatorname{Acyc}_{v}(Y)$.

Proposition 4.4. Let $Y$ be a connected graph. For any fixed $v \in \mathrm{v}[Y]$, there is a bijection

$$
\phi_{v}: \operatorname{Acyc}_{v}(Y) \longrightarrow \operatorname{Acyc}(Y) / \sim_{\kappa}
$$


Proof. Since $\kappa(Y)=\left|\operatorname{Acyc}(Y) / \sim_{\kappa}\right|=T_{Y}(1,0)=\left|\operatorname{Acyc}_{v}(Y)\right|$, it is sufficient to show that there is a surjection $\phi_{v}: \operatorname{Acyc}_{v}(Y) \longrightarrow \operatorname{Acyc}(Y) / \sim_{\kappa}$.

We first prove that each $A \in \operatorname{Acyc}(Y) / \sim_{\kappa}$ contains at least one element of $\operatorname{Acyc}_{v}(Y)$ by contradiction. Assume that $A \in \operatorname{Acyc}(Y) / \sim_{\kappa}$ contains no element of $\operatorname{Acyc}_{v}(Y)$, and choose $O_{Y} \in A$ such that $v$ is a source. Clearly, the assumption implies that there exists infinite length click-sequences from $O_{Y}$ not containing $v$. Let $c$ be such a click-sequence, and let $V^{\prime} \subset \mathrm{v}[Y]$ be the set containing all vertices that occur infinitely often in $c$. Then $V^{\prime} \neq \varnothing$, and since $v \notin V^{\prime}$ we have $\mathrm{v}[Y] \backslash V^{\prime} \neq \varnothing$. For such a click-sequence $c$ to exist there can be no edges of the form $\{s, t\} \in \mathrm{e}[Y]$ with $s \in V^{\prime}$ and $t \in \mathrm{v}[Y] \backslash V^{\prime}$, and we are forced to conclude that $Y$ is not connected, a contradiction. Hence any $A \in \operatorname{Acyc}(Y) / \sim_{\kappa}$ contains at least one element of $\operatorname{Acyc}_{v}(Y)$.

Non-equivalent $\kappa$-classes of $Y$ cannot have elements of $\operatorname{Acyc}_{v}(Y)$ in common, and since $\left|\operatorname{Acyc}(Y) / \sim_{\kappa}\right|=\left|\operatorname{Acyc}_{v}(Y)\right|$, we conclude that each $\kappa$-class of $Y$ contains a unique element of $\operatorname{Acyc}_{v}(Y)$. The map $\phi_{v}: \operatorname{Acyc}_{v}(Y) \longrightarrow \operatorname{Acyc}(Y) / \sim_{\kappa}$ defined by $\phi_{v}\left(O_{Y}\right)=\left[O_{Y}\right]$ is therefore a surjection and, by the initial comment, a bijection.

From Proposition 4.4 we immediately obtain:

Corollary 4.5. For any vertex $v$ of $Y$ the set $\operatorname{Acyc}_{v}(Y)$ is a transversal of $\operatorname{Acyc}(Y) / \sim_{\kappa}$.

The results in this section establish an alternative approach for deriving the recurrence in (1.1). However, our proof offers insight into the structure of the $\kappa$-classes, and it is our hope that this may lead to new techniques for studying conjugacy classes of Coxeter groups.

\section{ACKNOWLEDGEMENTS}

The first author would like to thank Jon McCammond for many helpful discussions. Both authors are grateful to the NDSSL group at Virginia Tech for their support of this research. Special thanks to Vic Reiner and William Y. C. Chen for valuable advice regarding the content, preparation, and structure of this paper. The work was partially supported by the Fields Institute in Toronto, Canada.

\section{REFERENCES}

[1] Anders Björner, László Lovász, and Peter Shor, Chip-firing games on graphs, European Journal of Combinatorics 12 (1991), 283-291. MR1120415 (92g:90193)

[2] B. Chen, Orientations, lattice polytopes, and group arrangements I. Chromatic and tension polynomials of graphs. Preprint at arXiv:0706.3273.

[3] Emeric Gioan, Enumerating degree sequences in digraphs and a cycle-cocycle reversing system, European Journal of Combinatorics 28 (2007), 1351-1366. MR 2305596 (2008a:05108)

[4] James E. Humphreys, Reflection groups and Coxeter groups, Cambridge Studies in Advanced Mathematics, no. 29, Cambridge University Press, 1990. MR1066460 (92h:20002)

[5] Matthew Macauley and Henning S. Mortveit, Cycle equivalence of graph dynamical systems, submitted. Preprint at arXiv:0802.4412.

[6] R. Marsh, M. Reineke, and A. Zelevinsky, Generalized associahedra via quiver representations, Transactions of the American Mathematical Society 355 (2003), 4171-4186. MR.1990581 (2004g:52014)

[7] Isabella Novik, Alexander Postnikov, and Bernd Sturmfels, Syzygies of oriented matroids, Duke Mathematical Journal 111 (2002), 287-317. MR1882136(2003b:13023)

[8] Peter Orlik and Hiroaki Terao, Arrangements of hyperplanes, A Series of Comprehensive Studies in Mathematics, Springer-Verlag, 1992. MR,1217488 (94e:52014) 
[9] C. M. Reidys, Acyclic orientations of random graphs, Advances in Applied Mathematics 21 (1998), no. 2, 181-192. MR.1634697(99e:05110)

[10] J. Y. Shi, The enumeration of Coxeter elements, Journal of Algebraic Combinatorics 6 (1997), 161-171. MR1436533 (98d:20048)

[11] Jian Yi Shi, Conjugacy relation on Coxeter elements, Advances in Mathematics 161 (2001), 1-19. MR1857933 (2002g:20072)

Department of Mathematics, University of California, Santa Barbara, Santa BarBara, California 93106-3080 - AND - NDSSL, Virginia Bioinformatics Institute, Virginia

Tech, Blacksburg, Virginia 24061

E-mail address: macauley@vt.edu

Current address: Department of Mathematical Sciences, Clemson University, Clemson, South Carolina 29634 - and - NDSSL, Virginia Bioinformatics Institute, Virginia Tech, Blacksburg, Virginia 24061

E-mail address: mmacaul@clemson.edu

Department of Mathematics, Virginia Tech, Blacksburg, Virginia 24061 - and NDSSL, Virginia Bioinformatics Institute, Virginia Tech, Blacksburg, Virginia 24061

E-mail address: henning.mortveit@vt.edu 